\title{
Enantioselective Synthesis of exo-4-Nitroprolinates from Nitroalkenes and Azomethine Ylides Catalyzed by Chiral Phosphoramidite-Silver(I) or Copper(II) Complexes
}

Luis M. Castelló, ${ }^{\mathrm{a}}$ Carmen Nájera, ${ }^{\mathrm{a}}$ José M. Sansano, ${ }^{\mathrm{a} *}$ Olatz Larrañaga, ${ }^{\mathrm{b}}$ Abel de Cózar, ${ }^{\mathrm{b}, \mathrm{c}}$ and Fernando P. Cossío ${ }^{\mathrm{b}, \mathrm{c}}$

\begin{abstract}
a Departamento de Química Orgánica, Instituto de Síntesis Orgánica (ISO), and Centro de Innovación en Química Avanzada (ORFEO-CINQA), Universidad de Alicante, Apdo. 99, E-03080-Alicante, Spain.

${ }^{\mathrm{b}}$ Departmento de Química Orgánica I, Facultad de Química, and Centro de Innovación en Química Avanzada (ORFEO CINQA), Universidad del País Vasco, P. K. 1072, E-20018 San Sebastián, Spain.

${ }^{\mathrm{c}}$ IKERBASQUE, Basque Foundation for Science, E-48011 Bilbao, Spain.
\end{abstract}

Fax: (+34)-965-903-549; phone: (+34)-965-903-549; e-mail: jmsansano@ua.es

Abstract: Chiral complexes formed by privileged phosphoramidites, from chiral binol and optically pure Davies' amines, and copper(II) triflate, silver(I) triflate or silver(I) benzoate are excellent catalysts for the general 1,3-dipolar cycloaddition between azomethine ylides generated from $\alpha$-amino acids derived imino esters and nitroalkenes. These three methods afford at room temperature high diastereoselectivityofexo-cycloadducts (4,5-trans-2,5-cis-4nitroprolinates) in high enantioselectivity. In general, the three procedures are complementary but silver catalysts are more versatile and less sensitive to sterically congested starting materials.

Procedure 1<smiles>[R]C=C[N+](=O)[O-]</smiles>
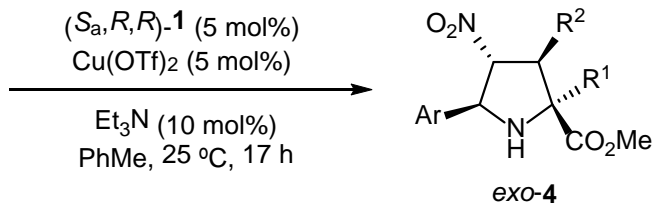<smiles>[R]C1[C@H]([N+](=O)[O-])[C@@H]([AlH2])N[C@]1([R])C(=O)O[Na]</smiles>

Procedure 2<smiles>[R]C=C[N+](=O)[O-]</smiles>
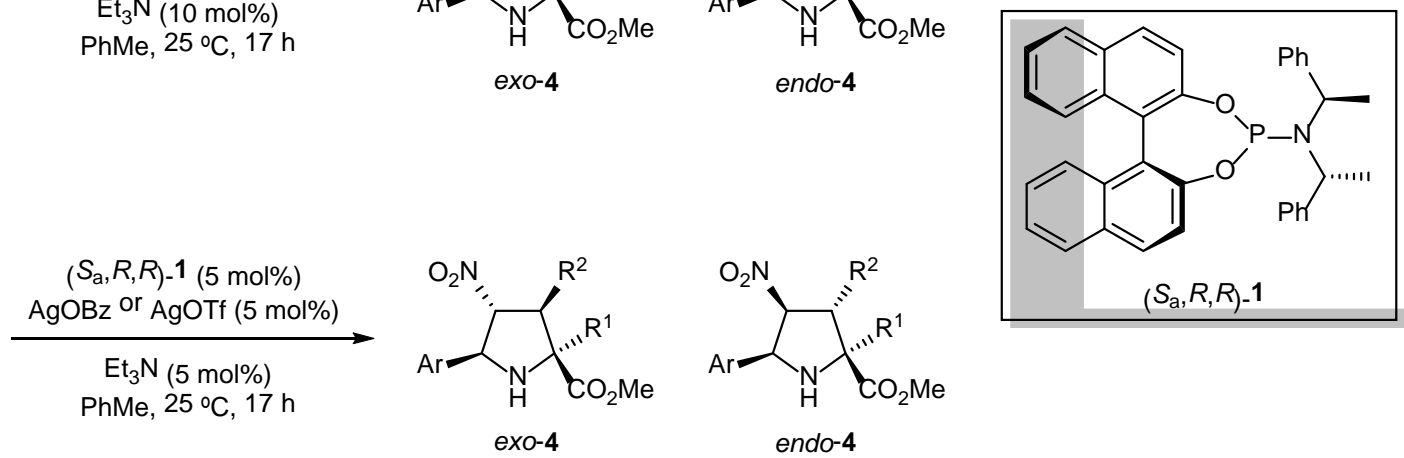

Scheme 1. General procedures for the synthesis of polysubstituted exo-nitroprolinates 4.

\section{Introduction}

The straightforward synthesis of enantiomerically enriched polysubstituted exo-4-nitroprolinates can be achieved through a 1,3-dipolar cycloaddition (1,3DC) ${ }^{1}$ between imino esters and nitroalkenes. ${ }^{2}$ The introduction of the versatile nitro group at the 4position of the pyrrolidine skeleton enhances the interest of these chiral molecules. Particularly,exo-4nitroprolinates with a specific 2,5-cis-4,5-transconfiguration have been used as leukocyte function associated antigen-1 antagonists during a cancer evolution, ${ }^{3}$ with important activity as inhibitors of $\alpha_{4} \beta_{1}$-integrin-mediated hepatic melanoma metastasis and even for treatments of other diseases. ${ }^{4}$ More simple exo-nitroprolinates such as $\mathbf{4}$ have shown a high efficiency as organocatalysts in asymmetric aldol reactions. ${ }^{5}$

For the construction of these exo-prolinates with 2,5cis-4,5-trans arrangement, $N$-arylideneamino esters have been employed as azomethine ylide precursors in the enantioselective 1,3-DC with nitroalkenes employing both organocatalysts and metal complexes as chiral catalysts. ${ }^{6}$ The major contributions using $\alpha$ - 
imino esters to get exo-adducts were achieved under the control of chiral metal complexes. ${ }^{7}$ Chiral Lewis acids formed by copper(I), copper(II), gold(I), and nickel(II) complexes were the most effective.2The only exception reported in all these examples is the employment of a copper(II) triflate-pyridyl bis(imidazolidine) as chiral complex, ${ }^{8}$ andvery interesting switchable chiral P,N-ferrocene ligands, ${ }^{9}$ which afforded the endo-adducts.

Taking in account that silver(I) is traditionally the most suitable cation to stabilize a metallodipole derived from iminoesters $3,^{10}$ any efficient $1,3-\mathrm{DC}$ with $\beta$-nitroalkenes has been reported. ${ }^{11}$ Only Fukuzawa's group published a highly effective asymmetric cycloaddition using benzophenonederived iminoglycinates and nitroalkenes catalyzed by silver(I)· thioclickferrophos complexes. ${ }^{12}$

Very recently, we have shown that phosphoramidites, ${ }^{13,14}$ derived from chiral binol-chiral Davies' amines, represented a matched combination for the enantioselective 1,3-dipolar cycloaddition (1,3DC) ${ }^{15}$ between nitroalkenes and azomethine ylides derived from imino esters. Cu(OTf) ${ }_{2}{ }^{16}$ and silver(I) salts $^{17}$ afforded exo-4-nitroprolinates in high diastereomeric ratios and enantioselectivities. Seminal works published in the literature concerning asymmetric 1,3-CD of imino esters and different dipolarophiles catalyzed by chiral phosphoramidite 1. silver(I)complexes supported this idea, ${ }^{18}$ obtaining, enantiomerically enriched polysubstituted endoproline derivatives in good chemical yields and excellent both diastereo- and enantioselections.

Herein, we wish to report a practical procedure for the synthesis ofexo-4-nitroprolinates $\mathbf{4}$ in which nitroalkenes underwent copper(II) and the less known silver(I)-catalyzed 1,3-dipolar cycloaddition with azomethine ylides obtained from $\alpha$-imino esters.

\section{Scope and Limitations}

The scope of the reaction was studied with different nitroalkenes $\mathbf{3}$, employing various arylideneimino esters 2 under the control of: a) the catalyst formed by phosphoramidite $\left(S_{\mathrm{a}}, R, R\right)-\mathbf{1}$ and $\mathrm{Cu}(\mathrm{OTf})_{2}$ (Scheme 1 , Procedure 1, Table 1); b)the catalyst originated by $\left(S_{\mathrm{a}}, R, R\right)-1$ and AgOTf (Scheme 1, Procedure 2, Table 1), and; c) the combination of phosphoramidite $\left(S_{\mathrm{a}}, R, R\right)-\mathbf{1}$ with AgOBz (Scheme 1 , Procedure 2, Table 1). Initially, the influence of the ester moiety of imines $\mathbf{2}$ was surveyed. Compound $\mathbf{4 a}$ was obtained as very clear crude reaction product and with the highest er in the presence of $\left(S_{\mathrm{a}}, R, R\right)$ 1. AgOBz complex (Table 1, entry 1). When the isopropyl ester of the corresponding dipole precursor was used, instead of the methyl ester, almost identical er for the reactions catalyzed by the two chiral silver complexes with a slightly better exo-diastereoselection for the isopropyl ester was obtained. The copper(II) catalyzed process gave better exo/endo and similar enantiomeric ratios. However, the lower yields(up to $70 \%$ ) of the exo-product $\mathbf{4 b}$ mixtures obtained in all of the reactions using the isopropyl ester obeyed to the appearance of a proportion of other unidentified diastereoisomers (ca. 20-28\% from crude product determined by ${ }^{1} \mathrm{H}$ NMR), (Table 1, entry 2). In consequence, the study of the scope of the reaction was next done with methyl $\alpha$-imino esters as metalloazomethine ylide precursors.

The study of the influence of the aryl substituent of the nitroalkene $\mathbf{3}$ for the 1,3-DC with methyl benzylidene glycinate revealed that, in general, higher chemical yields, exo-diastereoselectivities and enantioselections in products $4 \mathbf{c}-\mathbf{g}$ were observed when the reactions occurred in the presence of $\left(S_{\mathrm{a}}, R, R\right)-\mathbf{1} \cdot \mathrm{AgOBz}$ rather than with the silver(I) or copper(II) triflates(Table 1, entries 3-7). Only one exception was recorded when $m$-brominated nitrostyrene was employed as dipolarophile (Table 1, entry 6). In this example, $\left(S_{\mathrm{a}}, R, R\right)-\mathbf{1} \cdot \mathrm{Cu}(\mathrm{OTf})_{2}$ complex was the most effective catalyst giving the cleanest crude compound 4 f (90/10 dr, and 96:4 er). In the case of the heteroaromatic moiety bonded to the nitro component (2-furyl) was more appropriate with the chiral silver benzoate complex achieving $77 \%$ of chemical yield and $98 \%$ ee of the exo-4h (Table 2, entry 8). When the nitroalkene 3 beared an aliphatic cyclohexyl group a reversal diastereoselectivity was observed in the $\mathbf{1}$.AgOTf catalyzed 1,3-DC. The exclusive endo isomer was isolated in $71 \%$ yield but as a racemic mixture. However, an equimolar mixture of endo/exo diastereoisomers, in 75\% overall yield, was isolated in the analogous reaction performed with AgOBz. Again, the adduct endo-4i was separated as a racemate, whilst the exo-isomer $4 \mathbf{i}$ was obtained in low 32\% yield but with a $96 \%$ ee (Table 2, entry 9). On the other hand, the 1,3-DC with this nitroalkene3 $\left(\mathrm{R}^{2}=\mathrm{Cy}\right)$ failed with the $\mathrm{Cu}(\mathrm{OTf})_{2}$ complex.

When alanine, leucine, and phenylalanine derived imino esters $\mathbf{2}$ were used as azomethine ylide precursors, an increment of the endo-diastereoisomers $\mathbf{4 j - 1}$ was observed. This diastereoisomer was always obtained as a racemic mixture (Table 1, entries 1012). Alanine dipole precursor2 furnished very low proportions of exo-4j, which was isolated with high optical purity in silver-catalyzed processes (Table 1, entry 10). The other two quaternized pyrrolidines exo$\mathbf{4 k}$, and exo-4l were satisfactorily isolated ( $>99: 1 \mathrm{er})$ by employing $\left(S_{\mathrm{a}}, R, R\right)-\mathbf{1} \cdot \mathrm{Cu}(\mathrm{OTf})_{2}$ catalysis (Table 1 , entries 11 and 12).

With respect to the reaction of different methyl arylideneimino glycinates 2 with nitrostyrene, the more sterically hindered o-tolyl imino group also favoured the generation of the endo-isomer $\mathbf{4 m}$ but in less proportion in the case of AgOBz (Table 1, entry 13). For the $m$-tolyl imino group derivative the benzoate counteranion gave the highest 
enantioselections of compound exo-4n (Table 1, entry 14). It seems that the lower steric repulsion the higher proportion of exo-adduct and enantioselection. This general assumption can be supported by the transformations involving $p$-substituted arylidene imino esters2 (Table 2, entries 15-18). Thus, better results for compounds 4o-rwere observed when $\left(S_{\mathrm{a}}, R, R\right)$-1·AgOTf was the selected catalyst, rather than the processes mediated by the silver benzoate (Table 2, entries 15-18). Noticeably, compound exo4p could not be prepared through the copper(II)catalyzed method (Table 2, entry 16). Finally, in the reaction using substrate 2 with a 2-naphthyl substituent, productexo-4s was obtained with up to $72 \%$ de and 85\% ee when AgOTf was used better than $\mathrm{Cu}(\mathrm{OTf})_{2}$ and AgOBz (Table 2, entry 19).

Table 1.Scope of the diastereo- and enantioselective 1,3-DC between imino esters $\mathbf{2}$ and nitroalkenes $\mathbf{3}$ catalyzed by, $\left(S_{\mathrm{a}}, R, R\right)-\mathbf{1} \cdot \mathrm{Cu}(\mathrm{OTf})_{2},\left(S_{\mathrm{a}}, R, R\right)-\mathbf{1} \cdot \mathrm{AgOTf}$, and $\left(S_{\mathrm{a}}, R, R\right)-\mathbf{1} \cdot \mathrm{AgOBz}$.

\begin{tabular}{|c|c|c|c|c|c|c|c|c|c|c|c|}
\hline \multirow[b]{2}{*}{ Entry } & \multirow[b]{2}{*}{4} & \multirow[b]{2}{*}{ Structure } & \multicolumn{3}{|c|}{$\left(S_{\mathrm{a}}, R, R\right)-\mathbf{1} \cdot \mathrm{Cu}(\mathrm{OTf})_{2}$} & \multicolumn{3}{|c|}{$\left(S_{\mathrm{a}}, R, R\right)-\mathbf{1} \cdot \operatorname{AgOTf}$} & \multicolumn{3}{|c|}{$\left(S_{\mathrm{a}}, R, R\right)-\mathbf{1} \cdot \mathrm{AgOBz}$} \\
\hline & & & $\begin{array}{l}\text { exo/ } \\
\text { endo }{ }^{\mathrm{a}, \mathrm{b}}\end{array}$ & $\begin{array}{l}\text { Yield } \\
(\%)^{\mathrm{c}}\end{array}$ & $e r^{\mathrm{d}}$ & $\begin{array}{l}\text { exo/ } \\
e^{\text {endo }}{ }^{\mathrm{a}, \mathrm{b}}\end{array}$ & $\begin{array}{l}\text { Yield } \\
(\%)^{c}\end{array}$ & $e r^{\mathrm{d}}$ & $\begin{array}{l}\text { exo/ } \\
e^{\text {endo }}\end{array}$ & $\begin{array}{l}\text { Yield } \\
(\%)^{c}\end{array}$ & $e r^{\mathrm{d}}$ \\
\hline 1 & $4 a$ & & $89 / 11$ & 80 & $>99: 1$ & $90 / 10$ & 80 & $98: 2$ & $91 / 9$ & 88 & $>99: 1$ \\
\hline 2 & $4 b$ & & $99 / 1$ & 69 & $>99: 1$ & $98 / 2$ & 69 & $98: 2$ & $93 / 7^{\mathrm{b}}$ & 70 & $98: 2$ \\
\hline 3 & $4 c$ & & $82 / 18$ & 48 & 99:1 & $70 / 30$ & 52 & $95: 5$ & $93 / 7$ & 92 & 99:1 \\
\hline 4 & 4d & & $82 / 12$ & 73 & $96: 4$ & $89 / 11$ & 74 & $98: 2$ & $92 / 8$ & 88 & $>99: 1$ \\
\hline 5 & $4 e$ & & $73 / 27$ & 56 & $96: 4$ & $76 / 24$ & 64 & $97: 3$ & $91: 9^{b}$ & 76 & $96: 4$ \\
\hline 6 & $4 f$ & & $90 / 10$ & 61 & $94: 6$ & $81 / 19$ & 61 & $93: 7$ & $82 / 18^{b}$ & 61 & $94: 6$ \\
\hline 7 & $4 g$ & & $84 / 16$ & 70 & $95: 5$ & $85 / 15$ & 56 & 99:1 & $82 / 18$ & 77 & 99:1 \\
\hline 8 & $4 h$ & & $77 / 23$ & 41 & $91: 9$ & $68 / 32$ & 50 & $97: 3$ & $84 / 16$ & 77 & 99:1 \\
\hline 9 & $4 i$ & & - & - & - & $1 / 99$ & $71^{\mathrm{e}}$ & rac. & $50 / 50$ & $33^{d} 42^{e}$ & $98: 2^{f}$ \\
\hline 10 & $4 j$ & & $26 / 74$ & 18 & $r a c^{\mathrm{f}}$ & $21 / 79$ & $14^{\mathrm{d}} 63^{\mathrm{e}}$ & $93: 7^{f}$ & $27 / 73$ & $21^{\mathrm{d}} 69^{\mathrm{e}}$ & $96: 4^{\mathrm{f}}$ \\
\hline 11 & $4 k$ & & $92 / 8$ & 60 & $>99: 1^{\mathrm{f}}$ & $92 / 8$ & 46 & 98:2 & $35 / 65$ & $21^{\mathrm{d}} 53^{\mathrm{e}}$ & $99: 1^{f}$ \\
\hline
\end{tabular}




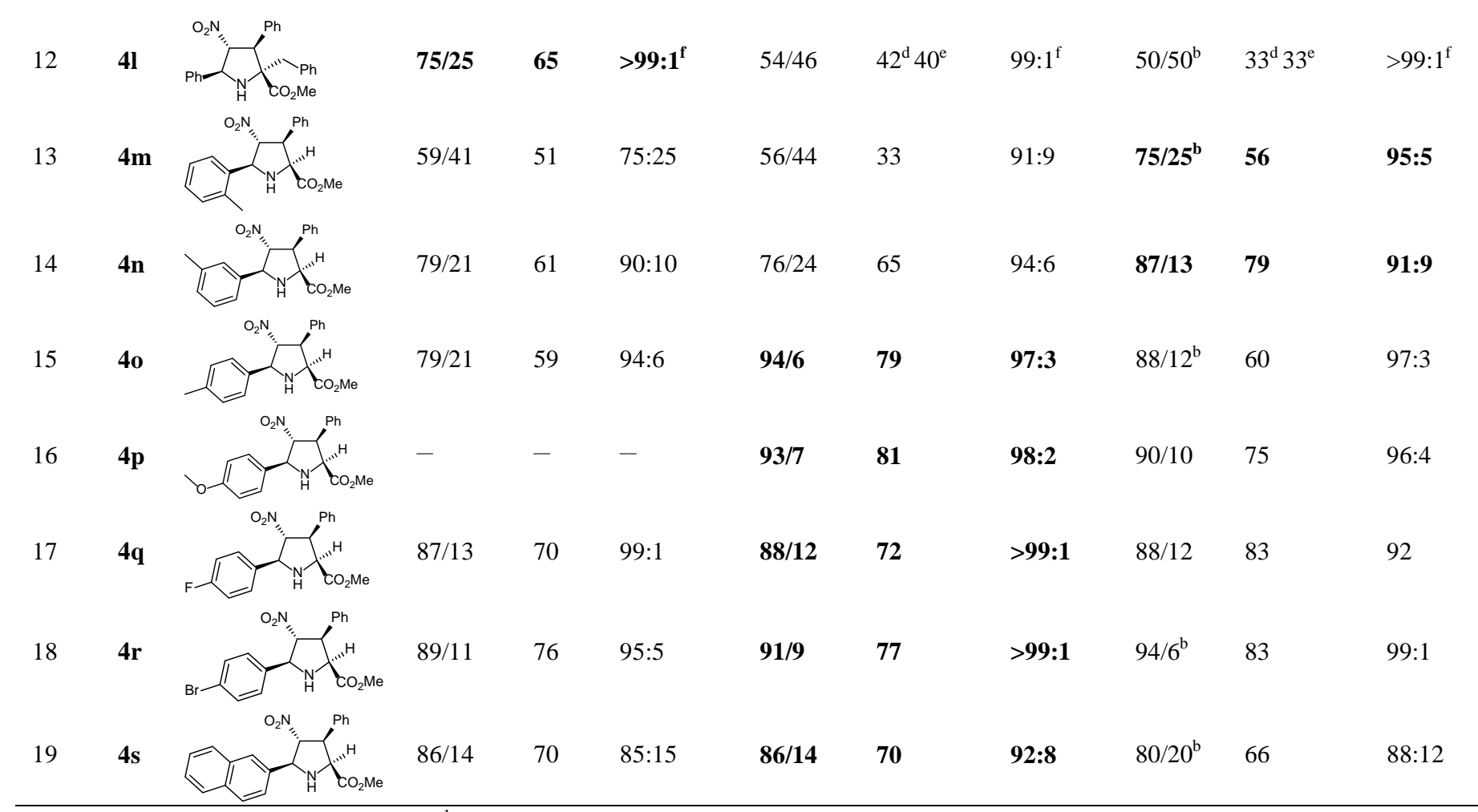

${ }^{\mathrm{a}}$ From the crude product, determined by ${ }^{1} \mathrm{H}$ NMR.

${ }^{\mathrm{b}}$ Other stereoisomers were detected in noticeable proportions.

${ }^{\mathrm{c}}$ Isolated yield (for the exo-adduct) after purification by flash chromatography.

${ }^{\mathrm{d}}$ For the exo-stereoisomer (HPLC).

e For de endo-isomer.

${ }^{\mathrm{f}}$ The endo-isomer was obtained as a racemic mixture.

In these efficient copper(II) or silver(I)-catalyzed cycloadditions of nitroalkenes,the influence of the position of the anion in the transition state is crucial to explain the diastereo- and enantioselectivity of the whole process because itself is able to block one of the two prochiral faces of the 1,3-dipole. Such as it can be deduced from DFT calculations, ${ }^{16,17}$ triflate anion is closer to the copper(II)centre than the corresponding silver(I) cation, whereas the distance silver(I) cation-dipole is shorter. These reasons justify the use of $\left(S_{a}, R, R\right)-\mathbf{1} \cdot \mathrm{Cu}(\mathrm{OTf})_{2}$ complex in the reactions involving $\alpha$-substituted imino esters2.

The influence of the benzoate anion vs. triflate anion can be explained by the assumption that benzoate (bulkier group than triflate) is oriented along the arylidene moiety of the dipole. Thus, a small $\pi$ stacking interaction is observed when $\mathbf{4}(\mathrm{R}=\mathrm{H}, \mathrm{Ar}=$ $\mathrm{Ph}$ ) is computed so, we think that a variation of this aromatic group implies that triflate anion is much less sensitive than the benzoate one. ${ }^{19}$ In this sense, the reaction regarding only $4(\mathrm{R}=\mathrm{H}, \mathrm{Ar}=\mathrm{Ph})$ and nitrostyrenes is optimally catalyzed by $\left(S_{\mathrm{a}}, R, R\right)$ 1. AgOBz complex. In contrast, when different aryl substituents are bonded to the 1,3-dipole precursor the employment of $\left(S_{\mathrm{a}}, R, R\right)-\mathbf{1} \cdot$ AgOTf complex is desirable.
In conclusion, an efficient preparation of enantiomerically enriched polysubstituted exo-4nitroprolinates has been achieved through a copper(II)- or silver(I) catalyzed 1,3-DC between imino esters and nitroalkenes. This method allows the preparation of these pyrrolidines in larger scale (0.5$0.8 \mathrm{~g})$ at room temperature. $\left(S_{\mathrm{a}}, R, R\right)-\mathbf{1} \cdot \mathrm{AgOBz}$ is the best catalyst to perform cycloadditions starting from methyl benzylideneglycinate. $\left(S_{\mathrm{a}}, R, R\right)-\mathbf{1} \cdot \mathrm{Cu}(\mathrm{OTf})_{2}$ complex is more suitable in the reactions involving $\alpha$ substituted imino esters 2 . Finally, $\left(S_{\mathrm{a}}, R, R\right)$-1·AgOTf catalyst is highly recommended when imines derived from aromatic aldehydes (different of benzaldehyde) are used.

\section{Procedures}

Anhydrous solvents were freshly distilled under an argon atmosphere and degasified by Freeze-Pump-Thaw methodology. Aldehydes were also distilled prior to use for the elaboration of the imino esters and nitroalquenes. They are very known molecules and are easily prepared. Copper salts were dried in a Kugel-Rorh apparatus and stored under an argon atmosphere. Melting points were determined with a Reichert Thermowar hot plate apparatus and are uncorrected. HPLC analyses were performed with a JASCO-2000 series equipped with a chiral column by using mixtures of $n$-hexane/isopropyl alcohol as the mobile phase at $25^{\circ} \mathrm{C}$. Yields refer to isolated 
compounds estimated to be $>95 \%$ pure as determined by ${ }^{1} \mathrm{H}$ NMR spectroscopy and HPLC analyses. All silver-mediated reactions were run avoiding light exposure.

Copper-Catalyzed Synthesis of exo-Cycloadducts 4. Procedure 1 (P1).

To a solution of the chiral phosphoramidite $(0.05 \mathrm{mmol})$ and $\mathrm{Cu}(\mathrm{OTf})_{2}(0.05 \mathrm{mmol})$ in dry toluene $(3 \mathrm{~mL})$ under argon atmosphere was added a solution of imino ester (1mmol) and nitroalkene $(1 \mathrm{mmol})$ in toluene $(5 \mathrm{~mL})$. To the resulting suspension triethylamine $(0.05 \mathrm{mmol}, 7 \mu \mathrm{L})$ was added and the mixture stirred at room temperature $\left(25^{\circ} \mathrm{C}\right)$ for $16-24 \mathrm{~h}$. The crude reaction mixture was filtered through a small celite path. The residue was purified by flash chromatography yielding pure exo-cycloadducts. Solid products were recrystallized inmixtures of n-hexane/ether. $\mathbf{4 a}$ and $\mathbf{4 f}$ were also prepared in 3 mmol scale obtaining identical results to the reported for the model reaction.

\section{Silver-Catalyzed Synthesis of exo-Cycloadducts 4.} Procedure 2 (P2-AgOTf) or (P2-AgOBz).

To a solution of the chiral phosphoramidite $\mathbf{1}(0.05 \mathrm{mmol})$ and AgX (0.05 mmol, triflate or benzoate) in dry toluene $(3 \mathrm{~mL})$ under argon atmosphere was added a solution of imino ester (1mmol) and nitroalkene $(1 \mathrm{mmol})$ in toluene $(5 \mathrm{~mL})$. To the resulting suspension triethylamine $(0.05 \mathrm{mmol}, 7 \mu \mathrm{L})$ was added and the mixture stirred at room temperature $\left(25^{\circ} \mathrm{C}\right)$. The crude reaction mixture was filtered through a small Celite path. The residue was purified by flash chromatography yielding pure exo-cycloadducts. Solid products were recrystallized in mixtures of $n$-hexane/ether. Compounds $\mathbf{4 a}$ and $\mathbf{4 f}$ were also prepared in $3 \mathrm{mmol}$ scale obtaining identical results to the reported for the model reaction.

(2S,3S,4R,5S)-Methyl 4-nitro-3,5-diphenylpyrrolidine-2carboxylate (4a)

According to P2-AgOBz, colorless needles, $n$-hexane/Et ${ }_{2} \mathrm{O}$ 4:1. mp: $104-105^{\circ} \mathrm{C}$.

$[\alpha]_{\mathrm{D}}{ }^{20}=128$ (c 1.1, $\mathrm{CHCl}_{3}$ ), >99:1 er; HPLC: (Daicel Chiralpak AS-H), 2-propanol/hexane 20:80, flow rate 0.4 $\mathrm{mL} / \mathrm{min}, \mathrm{t}_{\mathrm{R}}: 25.2 \mathrm{~min}$ (major) and $27 \mathrm{~min}$ (minor), $220 \mathrm{~nm}$;

IR (solid) $v_{\max }: 1265,1551,1731 \mathrm{~cm}^{-1}$.

${ }^{1} \mathrm{H}$ NMR: 2.71 (br s, 1H, NH) 3.26 (s, 3H, Me), 4.35 (t, $J=7.8$ $\mathrm{Hz}, 1 \mathrm{H}, \mathrm{CHCO}$ ), 4.48 (d, $J=9.1 \mathrm{~Hz}, 1 \mathrm{H}, \mathrm{CHPh}), 4.73$ (d, J = $7.8 \mathrm{~Hz}, 1 \mathrm{H}, \mathrm{CHPh}$ ), 5.19 (t, $J=8.0 \mathrm{~Hz}, 1 \mathrm{H}, \mathrm{CHNO}), 7.45-7.18$ (m, 8H, $\mathrm{ArH}$ ), 7.56-7.52 (m, 2H, $\mathrm{ArH}$ ).

${ }^{13} \mathrm{C}-\mathrm{NMR} \delta$ : $51.8\left(\mathrm{CH}_{3}\right), 53.7(\mathrm{CHPh}), 64.2(\mathrm{CHCO}), 67.5$ (PhCHNH), $95.0\left(\mathrm{CHNO}_{2}\right), 126.8,127.8,128.1,128.7,128.9$, 129.0, 135.8, 137.6 (ArC), $171.8(\mathrm{CO})$.

MS m/z: 326 (M+1, 0.1\%), 279 (16), 220 (73), 193 (100), 178 (12), 115 (42).

EA required for $\mathrm{C}_{18} \mathrm{H}_{18} \mathrm{~N}_{2} \mathrm{O}_{4}$ : C 66.3, $\mathrm{H}$ 5.6, $\mathrm{N}$ 8.6\%; found: C 66.4, H 5.3, N 8.6\%.

(2S,3R,4R,5S)-Isopropyl 4-nitro-3,5-diphenylpyrrolidine-2carboxylate (4b)

According to $\mathrm{P} 1$, colorless needles, $n$-hexane/Et ${ }_{2} \mathrm{O} 4: 1$.

mp: $130-132{ }^{\circ} \mathrm{C}$.

$[\alpha]_{\mathrm{D}}{ }^{20}=82.3\left(\right.$ c $\left.1.02 \mathrm{CHCl}_{3}\right)>99: 1$ er; HPLC (Daicel Chiralpak AD-H), 2-propanol/hexane 10:90, flow rate $1.0 \mathrm{~mL}$ $\min , \mathrm{t}_{\mathrm{R}} 15.5 \mathrm{~min}$ (major), $20.4 \mathrm{~min}$ (minor).
IR (solid) $v_{\max }: 1265,1551,1731 \mathrm{~cm}^{-1}$.

${ }^{1} \mathrm{H}$ NMR $\delta: 0.58\left(\mathrm{~d}, J=6.3 \mathrm{~Hz}, 3 \mathrm{H}, \mathrm{CH}_{3}\right), 1.06(\mathrm{~d}, J=6.3 \mathrm{~Hz}$, $3 \mathrm{H}, \mathrm{CH}_{3}$ ), 2.75 (br. s, $1 \mathrm{H}, \mathrm{NH}$ ), 4.37 (dd, $J=8.9,8.0 \mathrm{~Hz}, 1 \mathrm{H}$, CHPh), 4.46 (d, $J=9.1 \mathrm{~Hz}, 1 \mathrm{H}, \mathrm{CHCO}), 4.65$ [dt, $J=12.6,6.3$ $\mathrm{Hz}, 1 \mathrm{H}, \mathrm{CH}\left(\mathrm{CH}_{3}\right)_{2}$ ], 4.75 (d, $\left.J=8.3 \mathrm{~Hz}, 1 \mathrm{H}, \mathrm{PhCHN}\right), 5.20$ (dd, $J=8.3,8.3 \mathrm{~Hz}, 1 \mathrm{H}, \mathrm{CHNO}_{2}$ ), 7.19-7.30 (m, 5H, ArH), 7.36-7.45 (m, 3H, ArH), 7.54-7.57 (m, 2H, ArH).

${ }^{13} \mathrm{C}-\mathrm{NMR} \quad \delta: 20.7\left(\mathrm{CH}_{3}\right), 21.6\left(\mathrm{CH}_{3}\right), 53.5(\mathrm{CHPh}), 64.1$ (CHCO), $67.4\left[\mathrm{CH}\left(\mathrm{CH}_{3}\right)_{2}\right], 69.1(\mathrm{PhCHNH}), 95.4\left(\mathrm{CHNO}_{2}\right)$, 126.9, 128.1, 128.8, 128.9, 129.0, 129.4, 136.3, 137.6 (ArC), $170.8(\mathrm{CO})$.

MS m/z: $356\left(\mathrm{M}^{+}, 0.01 \%\right), 307$ (10), 220 (100), 193 (80), 115 (28).

HRMS required for $\left(\mathrm{C}_{20} \mathrm{H}_{22} \mathrm{~N}_{2} \mathrm{O}_{4}-\mathrm{NO}_{2}\right)$ : 309.4021; found 309.4028.

EA required for $\mathrm{C}_{20} \mathrm{H}_{22} \mathrm{~N}_{2} \mathrm{O}_{4}$ : C 67.8, $\mathrm{H} \mathrm{6.3,} \mathrm{N}$ 7.9\%; found: C 67.4, H 6.3, N 8.3\%.

(2S,3S,4R,5S)-Methyl pyrrolidine-2-carboxylate (4c).

4-nitro-5-phenyl-3-(p-tolyl)-

According to P2-AgOBz, colorless needles, $n$-hexane/Et ${ }_{2} \mathrm{O}$ 5:1. mp $108-110{ }^{\circ} \mathrm{C}$.

$[\alpha]_{\mathrm{D}}{ }^{20}=144.8\left(\right.$ c 1.0, $\left.\mathrm{CHCl}_{3}\right)$ 99:1 er. HPLC (Daicel Chiralpak AS-H), 2-propanol/n-hexane 5:95, flow rate $1.1 \mathrm{~mL} / \mathrm{min}, t_{R}$ $23.2 \mathrm{~min}$ (major), $24.2 \mathrm{~min}$ (minor).

IR (neat) $v_{\max }: 1212,1547,1736,2917 \mathrm{~cm}^{-1}$.

${ }^{1} \mathrm{H}$ NMR $\delta: 2.31$ (s, 3H, $\mathrm{CH}_{3} \mathrm{C}$ ), 2.75 (br. s, $1 \mathrm{H}, \mathrm{NH}$ ), 3.32 (s, $3 \mathrm{H}, \mathrm{OCH}_{3}$ ), 4.35 (dd, $\left.J=8.5 \mathrm{~Hz}, 1 \mathrm{H}, \mathrm{CHTol}\right), 4.48$ (d, $J=9$ $\mathrm{Hz}, 1 \mathrm{H}, \mathrm{CHCO}_{2} \mathrm{Me}$ ), 4.76 (d, $J=8.3 \mathrm{~Hz}, 1 \mathrm{H}, \mathrm{CHPh}$ ), 5.21 (dd, $\left.J=8.2,8.2 \mathrm{~Hz}, 1 \mathrm{H}, \mathrm{CHNO}_{2}\right), 7.13(\mathrm{~m}, 2 \mathrm{H}, \mathrm{ArH}), 7.40-7.45$ (m, 4H, $\operatorname{ArH}$ ), 7.55-7.57 (m, 3H, $\operatorname{ArH}$ ).

${ }^{13} \mathrm{C}-\mathrm{NMR} \delta: 21.0\left(\mathrm{CH}_{3} \mathrm{C}\right) 51.9\left(\mathrm{CHC}_{6} \mathrm{H}_{4} \mathrm{Me}\right), 53.5\left(\mathrm{CH}_{3}\right)$, $64.2\left(\mathrm{CHCO}_{2} \mathrm{Me}\right), 67.5(\mathrm{CHPh}), 95.1\left(\mathrm{CHNO}_{2}\right), 126.9$ (CHCHCHC), 127.7, 129.0, 129.1, 129.4 (ArC), 132.53 $\left(\mathrm{CH}_{3} \mathrm{C}\right), 137.52$ (CCHNH), 137.92 (CCHCHNH), 171.86 (CO).

MS m/z $294\left(\mathrm{M}^{+}-\mathrm{NO}_{2}, 9 \%\right), 234$ (36), 207 (100), 129 (15).

HRMS required for $\left(\mathrm{C}_{19} \mathrm{H}_{20} \mathrm{~N}_{2} \mathrm{O}_{4}-\mathrm{NO}_{2}\right)$ : 294.1400; found: 294.1399.

EA required for $\mathrm{C}_{19} \mathrm{H}_{20} \mathrm{~N}_{2} \mathrm{O}_{4}$ : C 67.0, H 5.9, $\mathrm{N}$ 8.2\%; found: C 67.3, H 6.2, N 7.9\%.

\section{$(2 S, 3 S, 4 R, 5 S)-$ Methyl 3-(4-fluorophenyl)-4-nitro-5-} phenylpyrrolidine-2-carboxylate (4d).

According to P2-AgOBz, colorless prisms, $n$-hexane/Et ${ }_{2} \mathrm{O}$ 5:1. mp 96.2-97 ${ }^{\circ} \mathrm{C}$.

$[\alpha]_{\mathrm{D}}{ }^{20}=108.1$ ( с $\left.1, \mathrm{CHCl}_{3}\right)>99: 1$ er; HPLC (Daicel Chiralpak AD-H), 2-propanol $/ n$ - hexane $10: 90$, flow rate $1.0 \mathrm{~mL} / \mathrm{min}, t_{R}$ 21.0 min (minor), 28.2 min (major).

IR (solid) $v_{\max }: 1265,1511,1553,1746 \mathrm{~cm}^{-1}$.

${ }^{1} \mathrm{H}$ NMR $\delta 2.58$ (br. s, $\left.1 \mathrm{H}, \mathrm{NH}\right), 3.34\left(\mathrm{~s}, 3 \mathrm{H}, \mathrm{OCH}_{3}\right), 4.34-4.44$ (m, 1H, CHCHCO), 4.51 (d, $J=9.0 \mathrm{~Hz}, 1 \mathrm{H}, \mathrm{CHCO}_{2} \mathrm{Me}$ ), 4.78 (d, $J=8.2 \mathrm{~Hz}, 1 \mathrm{H}, \mathrm{CHPh}), 5.16(\mathrm{dd}, J=8.0,8.0 \mathrm{~Hz}, 1 \mathrm{H}$, $\mathrm{CHNO}_{2}$ ), 6.99-7.11 (m, 2H, ArH), 7.23-7.32 (m, 2H, ArH), 7.36-7.49 (m, 3H, $\mathrm{ArH}$ ), 7.58 (d, $J=7.5 \mathrm{~Hz}, 2 \mathrm{H}, \mathrm{ArH})$.

${ }^{13} \mathrm{C}$ NMR $\delta: 51.9$ (CHCHCO), $52.8\left(\mathrm{CH}_{3}\right), 64.0$ (CHCO), 67.3 (CHPh), $95.2\left(\mathrm{CHNO}_{2}\right), 126.8,128.9,129.5,129.6,131.8$, 137.6, 161.2, 163.4 (ArC), 171.6 (CO).

MS $m /: z 298\left(\mathrm{M}^{+}-\mathrm{NO}_{2}, 10 \%\right), 238$ (55), 211 (100), 133 (19), 117 (18). 
HRMS required for $\left(\mathrm{C}_{18} \mathrm{H}_{17} \mathrm{FN}_{2} \mathrm{O}_{4}-\mathrm{NO}_{2}\right)$ : 298.1181; found: 298.1175.

EA required for $\mathrm{C}_{18} \mathrm{H}_{17} \mathrm{~N}_{2} \mathrm{O}_{4}$ : C 62.8, $\mathrm{H}$ 5.0, $\mathrm{N}$ 8.1\%; found: C 62.5, H 5.2, N 7.9\%.

\section{(2S,3S,4R,5S)-Methyl 3-(2-bromophenyl)-4-nitro-5-} phenylpyrrolidine-2-carboxylate (4e).

According to P2-AgOBz, pale yellow oil.

$[\alpha]_{\mathrm{D}}{ }^{20}=57.7$ (c $1.0 \mathrm{CHCl}_{3}$ ) 95:5 er, HPLC (Daicel Chiralpak AD-H), 2-propanol/n-hexane 10:90, flow rate $1.0 \mathrm{~mL} / \mathrm{min}, t_{R}$ 20.1 min (major), 23.9 min (minor).

IR (neat) $v_{\max }: 1549,1736,2926 \mathrm{~cm}^{-1}$.

${ }^{1} \mathrm{H}$ NMR $\delta 2.53$ (br. s, $1 \mathrm{H}, \mathrm{NH}$ ), 3.29 (s, 3H, $\mathrm{CH}_{3}$ ), 4.70 (d, $J=$ $9.1 \mathrm{~Hz}, 1 \mathrm{H}, \mathrm{CHPh}), 4.83\left(\mathrm{~d}, J=8.6 \mathrm{~Hz}, 1 \mathrm{H}, \mathrm{CHCO}_{2} \mathrm{Me}\right), 4.98$ (dd, $J=9.3,9.3 \mathrm{~Hz}, 1 \mathrm{H}, \mathrm{CHC}_{6} \mathrm{H}_{4} \mathrm{Br}$ ), 5.30 (dd, $J=9.0,9.0 \mathrm{~Hz}$, $1 \mathrm{H}, \mathrm{CHNO}_{2}$ ), 7.17 (dd, $J=7.7,1.4 \mathrm{~Hz}, 1 \mathrm{H}, \mathrm{ArH}$ ), 7.36-7.45 (m, 6H, ArH), 7.55-7.63 (m, 2H, ArH).

${ }^{13} \mathrm{C}$ NMR $\delta: 51.9$ (CHAr), $52.2\left(\mathrm{CH}_{3}\right), 61.9\left(\mathrm{CHCO}_{2} \mathrm{Me}\right), 67.2$ (CHPh), $92.9\left(\mathrm{CHNO}_{2}\right), 127.1(\mathrm{CBr}), 127.2,127.8,128.6$, 128.7, 129.1, 129.2, 133.3, 134.6, 137.3 (ArC), 172.2 (CO).

MS m/z 360 ( $\left.\mathrm{M}^{+}-\mathrm{NO}_{2}, 28 \%\right), 358$ (28), 347 (11), 345 (11), 300 (96), 298 (100), 273 (94), 271 (96), 219 (36), 192 (92).

HRMS required for $\left(\mathrm{C}_{18} \mathrm{H}_{17} \mathrm{BrN}_{2} \mathrm{O}_{4}-\mathrm{NO}_{2}\right)$ : 359.0338; found: 359.0343.

EA required for $\mathrm{C}_{18} \mathrm{H}_{17} \mathrm{BrN}_{2} \mathrm{O}_{4}$ : C 53.3, $\mathrm{H}$ 4.2, N 6.9\%; found C 53.1, H 4.1, N 6.5\%.

\section{(2S,3S,4R,5S)-Methyl} phenylpyrrolidine-2-carboxylate (4f).

According to $\mathrm{P} 1$, pale yellow prisms, $n$-hexane/Et ${ }_{2} \mathrm{O} 5: 1$. mp $72-74{ }^{\circ} \mathrm{C}$.

$[\alpha]_{\mathrm{D}}{ }^{20}=60.5\left(c\right.$ 0.85, $\left.\mathrm{CHCl}_{3}\right)$ 94:6er. HPLC (Daicel Chiralpak AD-H), 2-propanol $/ n$-hexane $5: 95$, flow rate $1.0 \mathrm{~mL} / \mathrm{min}, t_{R}$ 41.5 min (minor), 44.3 min (major).

IR (solid) $v_{\max }: 1547,1735,2928 \mathrm{~cm}^{-1}$.

${ }^{1} \mathrm{H}$ NMR $\delta: 2.73$ (br. s, $1 \mathrm{H}, \mathrm{NH}$ ), 3.38 (s, 3H, $\mathrm{CH}_{3}$ ), 4.33 (dd, $\left.1 \mathrm{H}, J=8.8,7.8 \mathrm{~Hz}, \mathrm{CHC}_{6} \mathrm{H}_{4} \mathrm{Br}\right), 4.50(\mathrm{~d}, J=8.9 \mathrm{~Hz}, 1 \mathrm{H}$, $\mathrm{CHCO}_{2} \mathrm{Me}$ ), 4.75 (d, $\left.J=8.1 \mathrm{~Hz}, 1 \mathrm{H}, \mathrm{CHPh}\right), 5.13$ (dd, $J=7.9$, $7.9 \mathrm{~Hz}, 1 \mathrm{H}, \mathrm{CHNO}_{2}$ ), 7.20-7.22 (m, 2H, ArH), 7.41-7.55 (m, 5H, $\mathrm{ArH}$ ), 7.55-7.57 (m, 2H, ArH).

${ }^{13} \mathrm{C}$ NMR $\delta: 52.0\left(\mathrm{CHC}_{6} \mathrm{H}_{4} \mathrm{Br}\right), 53.0\left(\mathrm{CH}_{3}\right), 64.1\left(\mathrm{CHCO}_{2} \mathrm{Me}\right)$, $67.4(\mathrm{CHPh}), 94.9\left(\mathrm{CHNO}_{2}\right), 122.8(\mathrm{CBr}), 126.3,126.9,129.1$, 129.1, 130.3, 131.2, 131.4, 137.5, 138.4 (ArC), 171.5 (CO).

MS m/z: $360\left(\mathrm{M}^{+}-\mathrm{NO}_{2}, 17 \%\right), 358$ (17), 298 (86), 270 (77), 192 (100), 117 (76).

HRMS required for $\left(\mathrm{C}_{18} \mathrm{H}_{17} \mathrm{BrN}_{2} \mathrm{O}_{4}-\mathrm{NO}_{2}\right)$ : 359.0338; found 359.0334 .

EA required for $\mathrm{C}_{18} \mathrm{H}_{17} \mathrm{BrN}_{2} \mathrm{O}_{4}$ : C 53.3, $\mathrm{H} \mathrm{4.2,} \mathrm{N} \mathrm{6.9 \% ;} \mathrm{found}$ C 52.9, H 4.1, N 6.6\%.

\section{(2S,3S,4R,5S)-Methyl}

\section{phenylpyrrolidine-2-carboxylate (4g).}

According to P2-AgOBz, colorless prisms, $n$-hexane/ $/ \mathrm{Et}_{2} \mathrm{O}$ 4:1. mp $89-91^{\circ} \mathrm{C}$.

$[\alpha]_{\mathrm{D}}{ }^{20}=96.0$ (c 0.7, $\mathrm{CHCl}_{3}$ ), 99:1er. HPLC (Daicel Chiralpak AD-H), 2-propanol $/ n$-hexane $10: 90$, flow rate $1.0 \mathrm{~mL} / \mathrm{min}, t_{R}$ 19.4 min (major), 26.8 min (minor).

IR (solid) $v_{\max }: 1544,1737,2925 \mathrm{~cm}^{-1}$.
${ }^{1} \mathrm{H}$ NMR $\delta: 2.75$ (br. s, $1 \mathrm{H}, \mathrm{NH}$ ), 3.34 (s, 3H, $\mathrm{CH}_{3}$ ), 4.21-4.33 (m, $1 \mathrm{H}, \mathrm{CHC}_{6} \mathrm{H}_{4} \mathrm{Br}$ ), 4.48 (d, $J=8.9 \mathrm{~Hz}, 1 \mathrm{H}, \mathrm{CHCO}_{2} \mathrm{Me}$ ), 4.73 (d, $J=8.1 \mathrm{~Hz}, 1 \mathrm{H}, \mathrm{CHPh}$ ), 5.08 (dd, $J=7.8,7.8 \mathrm{~Hz}, 1 \mathrm{H}$, $\mathrm{CHNO}_{2}$ ), 7.12-7.14 (m, 2H, ArH), 7.37-7.44 (m, 5H, ArH), 7.50-7.51 (m, 2H, $\mathrm{ArH})$.

${ }^{13} \mathrm{C}$ NMR $\delta: 52.0\left(\mathrm{CHC}_{6} \mathrm{H}_{4} \mathrm{Br}\right), 52.9\left(\mathrm{CH}_{3}\right), 64.0\left(\mathrm{CHCO}_{2} \mathrm{Me}\right)$, $67.3(\mathrm{CHPh}), 95.1\left(\mathrm{CHNO}_{2}\right), 122.3(\mathrm{CBr}), 126.8,129.0,129.1$, 129.6, 132.0, 135.3, 137.6 (ArC), 171.5 (CO).

MS m/z: $360\left(\mathrm{M}^{+}-\mathrm{NO}_{2}, 15 \%\right), 359$ (15), 300 (52), 298 (51), 273 (99), 271 (100), 219 (26), 192 (89), 117 (43), 115 (26).

HRMS required for $\left(\mathrm{C}_{18} \mathrm{H}_{17} \mathrm{BrN}_{2} \mathrm{O}_{4}-\mathrm{NO}_{2}\right)$ : 359.0338 , found 359.0330 .

EA required for $\mathrm{C}_{18} \mathrm{H}_{17} \mathrm{BrN}_{2} \mathrm{O}_{4}$ : C 53.3, $\mathrm{H}$ 4.2, N 6.9\%; found: C 52.9, H 4.2, N 6.6\%.

(2S,3S,4R,5S)-Methyl

3-(furan-2-yl)-4-nitro-5phenylpyrrolidine-2-carboxylate (4h).

According to P2-AgOBz, yellow prisms $n$-hexane/ $\mathrm{Et}_{2} \mathrm{O}$ 3:1. mp 60-62 ${ }^{\circ} \mathrm{C}$.

$[\alpha]_{\mathrm{D}}{ }^{20}=89.0$ (c 1, $\mathrm{CHCl}_{3}$ ) 99:1 er. HPLC (Daicel Chiralpak AD-H), 2-propanol $/ n$-hexane $10: 90$, flow rate $1.0 \mathrm{~mL} / \mathrm{min}, t_{R}$ $15.4 \mathrm{~min}$ (major), $18.8 \mathrm{~min}$ (minor).

IR (neat) $v_{\max }: 1209,1549,1740,2341,2359 \mathrm{~cm}^{-1} .{ }^{1} \mathrm{H}$ NMR $\delta$ : 2.77 (br. s, $1 \mathrm{H}, \mathrm{NH}$ ), 3.51 (s, 3H, $\mathrm{OCH}_{3}$ ), 4.40 (d, $J=8.5 \mathrm{~Hz}$, $1 \mathrm{H}, \mathrm{CHCO}$ ), 4.50 (dd, $J=8.4,7.2 \mathrm{~Hz}, 1 \mathrm{H}$, CHfuryl), 4.65 (d, $J$ $=8.0 \mathrm{~Hz}, 1 \mathrm{H}, \mathrm{CHPh}$ ), 5.23 (dd, $J=7.9,7.0 \mathrm{~Hz}, 1 \mathrm{H}, \mathrm{CHNO}_{2}$ ), 6.20 (d, $J=3.1 \mathrm{~Hz}, 1 \mathrm{H}, \mathrm{CCH}), 6.29$ (dd, $J=3.2,1.8 \mathrm{~Hz}, 1 \mathrm{H}$, CCHCHCHO), 7.25-7.61 (m, 6H, $\mathrm{ArH}$ and OCH).

${ }^{13} \mathrm{C}$ NMR $\delta: 47.9$ (CHfuryl), $52.2\left(\mathrm{CH}_{3}\right), 63.2(\mathrm{CHCO})$, 68.2(CHPh), $93.6 \quad\left(\mathrm{CHNO}_{2}\right), \quad 108.4 \quad(\mathrm{CCH}), \quad 110.5$ (CСHCHCHO), 126.8, 128.6, 128.9, 137.2 (ArC), 142.8 (OCH), 148.9 (OC), $171.1(\mathrm{CO})$.

MS m/z $270\left(\mathrm{M}^{+}-\mathrm{NO}_{2}, 12 \%\right), 183(100), 155(10), 117$ (12).

HRMS required for $\left(\mathrm{C}_{16} \mathrm{H}_{16} \mathrm{~N}_{2} \mathrm{O}_{5}\right)$ : 316.1059; found: 316.1049 .

(2S,3R,4S,5S)-Methyl-3-cyclohexyl-4-nitro-5phenylpyrrolidine-2-carboxylate (4i).

According to P2-AgOBz, white needles. $n$-hexane/ $\mathrm{Et}_{2} \mathrm{O}$ 4:1. mp $125-127^{\circ} \mathrm{C}$.

$[\alpha]_{\mathrm{D}}{ }^{20}=0.2\left(\right.$ c $\left.1,0 \mathrm{CHCl}_{3}\right)$ 52:48 er. HPLC (Chiralpak AD-H, 2-propanol $10 \%$ in hexane, flow $1.0 \mathrm{~mL} \mathrm{~min}, 14.5 \mathrm{~min}$ (minor), 16.2 min (major).Exo-4i (Chiralpak AD-H, 2-propanol $10 \%$ in hexane, flow $1.0 \mathrm{~mL}$ min, $17.1 \mathrm{~min}$ (major), $19.0 \mathrm{~min}$ (minor).

IR (neat): 1202, 1544, 1737, $2927 \mathrm{~cm}^{-1} .{ }^{1} \mathrm{H}-\mathrm{NMR}(400 \mathrm{MHz}$, $\left.\mathrm{CDCl}_{3}\right) \delta: 1.25-1.32\left(\mathrm{~m}, 5 \mathrm{H}, \mathrm{CH}_{2}\right), 1.58-1.67[\mathrm{~m}, 1 \mathrm{H}$, $\mathrm{CH}\left(\mathrm{CH}_{2}\right)_{2}$ ], 1.72-1.86 (m, 5H, $\left.\mathrm{CH}_{2}\right), 2.87$ (td, $J=7,2.4 \mathrm{~Hz}$, $\left.1 \mathrm{H}, \mathrm{CHCH}\left(\mathrm{CH}_{2}\right)_{2}\right), 3.28$ (dd, $\left.J=11.5,10.7 \mathrm{~Hz}, 1 \mathrm{H}, \mathrm{NH}\right), 3.83$ (m, $1 \mathrm{H}, \mathrm{CHCO}_{2} \mathrm{Me}$ ), 4.47 (dd, $J=12.3,5.9 \mathrm{~Hz}, 1 \mathrm{H}, \mathrm{CHPh}$ ), 5.11(dd, $J=5.9,2.4 \mathrm{~Hz}, 1 \mathrm{H}, \mathrm{CHNO}_{2}$ ), 7.26-7.35 (m, 5H, ArH). ${ }^{13} \mathrm{C}-\mathrm{NMR}\left(75 \mathrm{MHz}, \mathrm{CDCl}_{3}\right) \delta: 26.13\left(\mathrm{CH}_{2} \mathrm{CH}_{2} \mathrm{CH}_{2} \mathrm{CH}\right), 2 \mathrm{x}$ 29.94 $\left(\mathrm{CH}_{2} \mathrm{CH}_{2} \mathrm{CH}_{2} \mathrm{CH}\right), 2 \times 31.51\left(\mathrm{CH}_{2} \mathrm{CH}_{2} \mathrm{CH}_{2} \mathrm{CH}\right), 39.73$ $\left(\mathrm{CH}_{2} \mathrm{CH}_{2} \mathrm{CH}_{2} \mathrm{CH}\right), \quad 52.65$ (CHCy), $57.09\left(\mathrm{CH}_{3}\right), 63.10$ $\left(\mathrm{CHCO}_{2} \mathrm{Me}\right), 67.73(\mathrm{CHPh}), 93.33\left(\mathrm{CHNO}_{2}\right), 126.17,128.46$, 128.65, 134.26 ( $\mathrm{ArC}), 172.38$ (CO). MS m/z ( $\left.\mathrm{M}^{+}-\mathrm{NO}_{2}\right) 285$ (13), 273 (16), 226 (82), 144 (100),117 (28). HRMS required for $\left(\mathrm{C}_{18} \mathrm{H}_{24} \mathrm{~N}_{2} \mathrm{O}_{4}\right)$ : 332.3934; found: 332.3927 . 


\section{(2S,3S,4S,5S)-Methyl} diphenylpirrolidine-2-carboxylate $(4 \mathbf{j})$.

According to P2-AgOTf, yellow oil.

$[\alpha]_{\mathrm{D}}{ }^{20}=-10.7\left(\mathrm{c} 1, \mathrm{CHCl}_{3}\right)$ 55:45 er. HPLC (Chiralpak AD-H, 2-propanol $10 \%$ in hexane, flow $1.0 \mathrm{~mL}$ min, $9.3 \mathrm{~min}$ (minor), 15.6 min (major). Exo-4j(Chiralpak AD-H, 2-propanol 10\% in hexane, flow $1.0 \mathrm{~mL} \mathrm{~min}, 9.1 \mathrm{~min}$ (minor), $17.4 \mathrm{~min}$ (major).

IR (neat): 1257, 1549, $1731 \mathrm{~cm}^{-1} .{ }^{1} \mathrm{H}-\mathrm{NMR}$ (300 MHz, $\mathrm{CDCl}_{3}$ ) $\delta: 1.19\left(\mathrm{~s}, 3 \mathrm{H}, \mathrm{CCH}_{3}\right), 3.25(\mathrm{br}, 1 \mathrm{H}, \mathrm{NH}), 3.86(\mathrm{~s}, 3 \mathrm{H}$, $\mathrm{CO}_{2} \mathrm{CH}_{3}$ ), $4.52(\mathrm{~d}, J=6.1 \mathrm{~Hz}, 1 \mathrm{H}, \mathrm{CHC}), 5.08$ (d, $J=7.4 \mathrm{~Hz}$, $1 \mathrm{H}, \mathrm{CHN}), 5.65\left(\mathrm{dd}, J=7.4,6.1 \mathrm{~Hz}, 1 \mathrm{H}, \mathrm{CHNO}_{2}\right.$ ), 7.23-7.49 (m, 10H, ArH). ${ }^{13} \mathrm{C}-\mathrm{NMR}\left(75 \mathrm{MHz}, \mathrm{CDCl}_{3}\right) \delta: 22.0\left(\mathrm{CCH}_{3}\right)$, $52.9(\mathrm{CHC}), 56.9\left(\mathrm{OCH}_{3}\right), 64.9(\mathrm{CHN}), 68.8\left(\mathrm{CCH}_{3}\right), 95.6$ $\left(\mathrm{CHNO}_{2}\right), 126.8(\mathrm{ArC}), 128.0(\mathrm{ArC}), 128.5(\mathrm{ArC}), 128.6$ (ArC), 128.7 (ArC), 128.8(ArC), 135.3(ArC), 135.5(ArC), 174.7(CO). MS m/z ( $\left.\mathrm{M}^{+}-\mathrm{NO}_{2}\right)$ 281(45), 234(100), 219(17), 193(37), 115(21). HRMS required for $\left(\mathrm{C}_{19} \mathrm{H}_{20} \mathrm{~N}_{2} \mathrm{O}_{4}\right)$ : 340.1422, found: 281.1428 .

(2S, 3R, 4R, 5S)-Methyl 2-isobutyl-4-nitro-3,5diphenylpyrrolidine-2-carboxylate (4k).

According to $\mathrm{P} 1$, colorless prisms, $n$-hexane/Et ${ }_{2} \mathrm{O} 4: 1$.

mp $105-107^{\circ} \mathrm{C}$.

$[\alpha]_{\mathrm{D}}{ }^{20}=59.0$ (c 1, $\mathrm{CHCl}_{3}$ ), 99:1 er; HPLC (Daicel Chiralpak AD-H), 2-propanol $/ n$-hexane 10:90, flow rate $1.0 \mathrm{~mL} / \mathrm{min}, t_{R}$ 9.9 min (major), $14.5 \mathrm{~min}$ (minor). IR (solid) $v_{\max }: 1230,1545$, $1730 \mathrm{~cm}^{-1}$.

${ }^{1} \mathrm{H}$ NMR $\delta: 0.81\left(\mathrm{~d}, J=6.7 \mathrm{~Hz}, 3 \mathrm{H}, \mathrm{CH}_{3}\right), 1.04(\mathrm{~d}, J=6.7 \mathrm{~Hz}$, $3 \mathrm{H}, \mathrm{CH}_{3}$ ), 1.57 (br. s, $1 \mathrm{H}, \mathrm{NH}$ ), 1.79 (td, $J=13.3,6.7 \mathrm{~Hz}, 1 \mathrm{H}$, $\mathrm{CH}$ ), 1.96 (dd, $J=13.9,5.7, \mathrm{~Hz}, 1 \mathrm{H}, \mathrm{CH}_{2}$ ), 2.15 (dd, $J=13.9$, $6.9 \mathrm{~Hz}, 1 \mathrm{H}, \mathrm{CH}_{2}$ ), 3.19 (s, 3H, $\mathrm{CH}_{3}$ ), 4.00 (d, $J=9.9 \mathrm{~Hz}, 1 \mathrm{H}$, CHC), 4.75 (d, $J=9.2 \mathrm{~Hz}, 1 \mathrm{H}, \mathrm{CHNH}$ ), 5.23 (dd, $J=9.2,9.2$ $\mathrm{Hz}, 1 \mathrm{H}, \mathrm{CHNO}_{2}$ ), 7.14-7.22 (m, 2H, $\mathrm{ArH}$ ), 7.25-7.47 (m, 6H, $\mathrm{ArH})$, 7.54-7.61 (m, 2H, $\mathrm{ArH})$.

${ }^{13} \mathrm{C}$ NMR $\delta: 22.8,24.3(2 \mathrm{xMe}), 25.6\left(\mathrm{CH}_{2}\right), 46.2\left(\mathrm{CHCH}_{2}\right)$, 51.8, 62.7 (2xCHPh), $66.1\left(\mathrm{CCO}_{2}\right), 73.2,94.9\left(\mathrm{CHNO}_{2}\right)$, 126.9, 127.7, 128.2, 128.6, 128.9, 129.1, 135.6, 137.2 (ArC), 174.5 (CO).

MS m/z: $337\left(\mathrm{M}^{+}-\mathrm{NO}_{2}, 25 \%\right), 193(100), 115(20)$.

HRMS required for $\left(\mathrm{C}_{22} \mathrm{H}_{26} \mathrm{~N}_{2} \mathrm{O}_{4}-\mathrm{NO}_{2}\right)$ : 321.1729; found: 321.1750. EA required for $\mathrm{C}_{22} \mathrm{H}_{26} \mathrm{~N}_{2} \mathrm{O}_{4}$ : C 69.1, H 6.9, N 7.3; found: C 69.5, H 6.8, N 7.4\%.

\section{(2S,3R,4R,5S)-Methyl}

diphenylpyrrolidine-2-carboxylate (4l).

According to P1, colorless oil.

$[\alpha]_{\mathrm{D}}{ }^{20}=25.2\left(\right.$ c 1,0 $\left.\mathrm{CHCl}_{3}\right)$, >99:1 er. HPLC (Daicel Chiralpak AD-H), 2-propanol $/ n$-hexane $10: 90$, flow rate $1.0 \mathrm{~mL} / \mathrm{min}, t_{R}$ $20.8 \mathrm{~min}$ (minor), $30.1 \mathrm{~min}$ (major).

IR (neat) $v_{\max }: 1208,1547,1723,2946 \mathrm{~cm}^{-1}$.

${ }^{1} \mathrm{H}$ NMR $\delta$ : 2.49 (br. s, $1 \mathrm{H}, \mathrm{NH}$ ), 3.22 (s, 3H, $\mathrm{OCH}_{3}$ ), 3.32 (d, $J$ $\left.=13.6 \mathrm{~Hz}, 1 \mathrm{H}, \mathrm{CH}_{2}\right), 3.42\left(\mathrm{~d}, J=13.5 \mathrm{~Hz}, 1 \mathrm{H}, \mathrm{CH}_{2}\right), 4.15(\mathrm{~d}$, $J=9.7 \mathrm{~Hz}, 1 \mathrm{H}, \mathrm{CHCCC}), 4.67$ (d, $J=9.0 \mathrm{~Hz}, 1 \mathrm{H}, \mathrm{CHPh}), 5.23$ (dd, $J=9.4,9.4 \mathrm{~Hz}, 1 \mathrm{H}, \mathrm{CHNO}_{2}$ ), 7.15-7.56 (m, $15 \mathrm{H}, \mathrm{ArH}$ ).

${ }^{13} \mathrm{C}$ NMR $\delta: 42.8\left(\mathrm{CH}_{2}\right), 51.9(\mathrm{CHPh}), 60.3\left(\mathrm{CH}_{3}\right), 65.8$ $(\mathrm{NCHPh}), 73.6(\mathrm{qC}), 95.1\left(\mathrm{CHNO}_{2}\right), 127.0,127.1,127.9$,
128.3, 128.3, 128.4, 129.0, 129.1, 130.5, 135.8, 136.0, 137.5 (ArC), $173.42(\mathrm{CO})$.

MS m/z: 326 (M+Bn, 19\%), 325 (97), 279 (20), 278 (100), 246 (76), 219 (41), 193 (20), 115(29).

HRMS required for $\left(\mathrm{C}_{25} \mathrm{H}_{24} \mathrm{~N}_{2} \mathrm{O}_{4}-\mathrm{CO}_{2} \mathrm{Me}\right)$ : 357.1608; found 357.1604 .

(2S,3S,4R,5S)-Methyl tolyl)pyrrolidine-2-carboxylate (4m).

4-nitro-3-phenyl-5-(2-

According to P2-AgOBz, pale yellow needles, $n$-hexane/Et ${ }_{2} \mathrm{O}$ 5:1.

mp $71-72{ }^{\circ} \mathrm{C}$.

$[\alpha]_{\mathrm{D}}{ }^{20}=108.2\left(c\right.$ 0.3, $\left.\mathrm{CHCl}_{3}\right)$ 95:5 er.HPLC (Daicel Chiralpak $\mathrm{AD}$-H), 2-propanol $/ n$-hexane $10: 90$, flow rate $1.0 \mathrm{~mL} / \mathrm{min}, \mathrm{t}_{\mathrm{R}}$ $20.4 \mathrm{~min}$ (minor), $25.3 \mathrm{~min}$ (major).

IR (solid) $v_{\max }: 1213,1548,1736 \mathrm{~cm}^{-1}$.

${ }^{1} \mathrm{H}$ NMR $\delta: 2.38$ (s, 3H, $\mathrm{CCH}_{3}$ ), 2.55 (br. s, $1 \mathrm{H}, \mathrm{NH}$ ), 3.28 (s, $3 \mathrm{H}, \mathrm{OCH}_{3}$ ), 4.43 (dd, $\left.J=10.1,7.1 \mathrm{~Hz}, 1 \mathrm{H}, \mathrm{CHPh}\right), 4.50$ (d, $J=$ $9.4 \mathrm{~Hz}, 1 \mathrm{H}, \mathrm{CHCO}$ ), 5.10 (d, $J=8.8 \mathrm{~Hz}, 1 \mathrm{H}, \mathrm{CHTol}$ ), 5.34 (dd, $J=8.7,8.7 \mathrm{~Hz}, 1 \mathrm{H}, \mathrm{CHNO}_{2}$ ), 7.20-7.37 (m, 9H, ArH).

${ }^{13} \mathrm{C}$ NMR $\delta: 19.2\left(\mathrm{CCH}_{3}\right), 51.8(\mathrm{CHPh}), 54.0\left(\mathrm{OCH}_{3}\right), 63.3$ (CHCO), 64.0 (CHTol), $93.8\left(\mathrm{CHNO}_{2}\right), 125.6,126.8,127.7$, 128.2, 128.6, 128.7, 130.9, 135.9, 135.6, 137.1 (ArC), 172.1 (CO).

MS m/z: $359\left(\mathrm{M}^{+}-\mathrm{NO}_{2}, 21 \%\right), 340$ (2), 293 (12), 234 (61), 207 (100), 191 (12), 131 (20), 115 (25).

EA required for $\mathrm{C}_{19} \mathrm{H}_{20} \mathrm{~N}_{2} \mathrm{O}_{4}$ : C 67.0, H 5.9, $\mathrm{N} 8.2 \%$; found C 66.9, H 5.5, N 8.4\%.

$(2 S, 3 S, 4 R, 5 S)-$ Methyl

4-nitro-3-phenyl-5-(3tolyl)pyrrolidine-2-carboxylate (4n).

According to P2-AgOBz, pale yellow prisms, $n$-hexane/Et ${ }_{2} \mathrm{O}$ 5:1.

mp 56-58 ${ }^{\circ} \mathrm{C}$.

$[\alpha]_{\mathrm{D}}{ }^{20}=99.8$ (c 0.74, $\mathrm{CHCl}_{3}$ ) 95:5 er(after recrystallization). HPLC (Daicel Chiralpak AD-H), 2-propanol/n-hexane 10:90, flow rate $1.0 \mathrm{~mL} / \mathrm{min}, \mathrm{t}_{\mathrm{R}} 15.1 \mathrm{~min}$ (minor), $19.9 \mathrm{~min}$ (major). IR (solid) $v_{\max }: 1213,1265,1549,1737 \mathrm{~cm}^{-1}$.

${ }^{1} \mathrm{H}$ NMR $\delta$ : 2.22 (br. s, $1 \mathrm{H}, \mathrm{NH}$ ), 2.39 (s, 3H, $\mathrm{CH}_{3} \mathrm{C}$ ), 3.30 (s, $3 \mathrm{H}, \mathrm{OCH}_{3}$ ), 4.40 (dd, $J=8.7,8.7 \mathrm{~Hz}, 1 \mathrm{H}, \mathrm{CHPh}$ ), 4.53 (d, $J=$ $9.2 \mathrm{~Hz}, 1 \mathrm{H}, \mathrm{CHCO}), 4.77$ (d, $J=8.5 \mathrm{~Hz}, 1 \mathrm{H}, \mathrm{CHTol}$ ), 5.26 (dd, $\left.J=8.4,8.4 \mathrm{~Hz}, 1 \mathrm{H}, \mathrm{CHNO}_{2}\right), 7.19-7.38$ (m, 9H, $\mathrm{ArH}$ ).

${ }^{13} \mathrm{C}$ NMR $\delta: 21.5\left(\mathrm{CCH}_{3}\right), 52.0(\mathrm{CHPh}), 53.7\left(\mathrm{OCH}_{3}\right), 64.0$ (CHCO), 67.4 (CHTol), $94.5\left(\mathrm{CHNO}_{2}\right), 123.9,127.6,127.8$, 128.2, 128.4, 128.8, 129.0, 129.4, 129.9, 138.9 (ArC), 171.6 (CO).

MS m/z: $294\left(\mathrm{M}^{+}-\mathrm{NO}_{2}, 21 \%\right), 234$ (75), 207 (100), 131 (20), 115 (29).

HRMS required for $\left(\mathrm{C}_{19} \mathrm{H}_{20} \mathrm{~N}_{2} \mathrm{O}_{4}-\mathrm{NO}_{2}\right)$ : 294.1572, found: 294.1565.

EA required for $\mathrm{C}_{19} \mathrm{H}_{20} \mathrm{~N}_{2} \mathrm{O}_{4}$ : C 67.0, H 5.9, N 8.2\%; found: C 67.2, H 6.0, N 8.2\%.

(2S,3S,4R,5S)-Methyl

4-nitro-3-phenyl-5-(4tolyl)pyrrolidine-2-carboxylate (4o).

According to P2-AgOTf, pale yellow prisms, $n$-hexane/ $\mathrm{Et}_{2} \mathrm{O}$ 8:1.

mp $90-92{ }^{\circ} \mathrm{C}$. 
$[\alpha]_{\mathrm{D}}{ }^{20}=81.9\left(c\right.$ 0.72, $\left.\mathrm{CHCl}_{3}\right)$ 97:3 er.HPLC (Daicel Chiralpak AD-H), 2-propanol $/ n$-hexane $10: 90$, flow rate $1.0 \mathrm{~mL} / \mathrm{min}, t_{R}$ $22.3 \mathrm{~min}$ (minor), $28.0 \mathrm{~min}$ (major).

IR (solid) $v_{\max }: 1220,1545,1725 \mathrm{~cm}^{-1}$.

${ }^{1} \mathrm{H}$ NMR $\delta: 2.30$ (s, 3H, $\mathrm{CH}_{3} \mathrm{C}$ ), 2.65 (br. s, $1 \mathrm{H}, \mathrm{NH}$ ), 3.21 (s, $3 \mathrm{H}, \mathrm{OCH}_{3}$ ), 4.29 (dd, $\left.J=9.0,9.0 \mathrm{~Hz}, 1 \mathrm{H}, \mathrm{CHPh}\right), 4.41$ (d, $J=$ $9.1 \mathrm{~Hz}, 1 \mathrm{H}, \mathrm{CHCO}$ ), 4.64 (d, $J=8.4 \mathrm{~Hz}, 1 \mathrm{H}, \mathrm{CHTol}$ ), 5.12 (dd, $\left.J=8.1,8.1 \mathrm{~Hz}, 1 \mathrm{H}, \mathrm{CHNO}_{2}\right), 7.14-7.38(\mathrm{~m}, 9 \mathrm{H}, \mathrm{ArH})$.

${ }^{13} \mathrm{C}$ NMR $\delta: 21.2\left(\mathrm{CCH}_{3}\right), 51.8(\mathrm{CHPh}), 53.8\left(\mathrm{OCH}_{3}\right), 64.2$ (CHCO), 67.5 (CHTol), $95.1\left(\mathrm{CHNO}_{2}\right), 126.9,127.8,128.1$, 128.8, 129.2, 129.7, 134.5, 135.9 (ArC), 171.9 (CO). MS m/z: $294\left(\mathrm{M}^{+}-\mathrm{NO}_{2}, 10 \%\right), 293$ (25), 234 (85), 207 (100), 191 (16), 131 (26), 115 (26).

HRMS required for $\left(\mathrm{C}_{19} \mathrm{H}_{20} \mathrm{~N}_{2} \mathrm{O}_{4}-\mathrm{NO}_{2}\right)$ : 294.1572; found: 294.1562.

EA required for $\mathrm{C}_{19} \mathrm{H}_{20} \mathrm{~N}_{2} \mathrm{O}_{4}$ : C 67.0, $\mathrm{H} \mathrm{5.9,} \mathrm{N}$ 8.2\%; found: C 66.8, H 5.8, N 7.9\%.

\section{(2S,3S,4R,5S)-Methyl 5-(4-methoxyphenyl)-4-nitro-3-} phenylpyrrolidine-2carboxylate (4p).

According to P2-AgOTf, yellow oil.

$[\alpha]_{\mathrm{D}}{ }^{20}=95.2\left(c 1.9, \mathrm{CHCl}_{3}\right)$ 98:2 er. HPLC (Chiralpak AD-H, 2-propanol $30 \%$ in hexane, flow $1.0 \mathrm{~mL} \mathrm{~min}, 19.9 \mathrm{~min}$ (minor), 25.2 min (major).

IR(neat): 1177, 1213, 1248, 1513, 1547, $1735 \mathrm{~cm}^{-1}$.

${ }^{1} \mathrm{H}-\mathrm{NMR}\left(300 \mathrm{MHz}, \mathrm{CDCl}_{3}\right.$ ) $\delta: 3.29\left(\mathrm{~s}, 3 \mathrm{H}, \mathrm{PhOCH}_{3}\right.$ ), 3.83 (s, $3 \mathrm{H}, \mathrm{CO}_{2} \mathrm{CH}_{3}$ ), 4.41 (dd, $\left.J=8.4,8.4 \mathrm{~Hz}, 1 \mathrm{H}, \mathrm{CHPh}\right), 4.50$ (d, $J$ $=9.2 \mathrm{~Hz}, 1 \mathrm{H}, \mathrm{CHCO}), 4.74(\mathrm{~d}, J=8.5 \mathrm{~Hz}, 1 \mathrm{H}, \mathrm{CHN}), 5.22$ (dd, $J=8.4,8.4 \mathrm{~Hz}, 1 \mathrm{H}, \mathrm{CHNO}_{2}$ ), 6.95 (d, $J=8.7 \mathrm{~Hz}, 2 \mathrm{H}$, ArH), 7.19-7.36 (m, 5H, ArH), 7.50 (d, $J=8.7 \mathrm{~Hz}, 2 \mathrm{H})$.

${ }^{13} \mathrm{C}$ NMR (101 MHz, $\left.\mathrm{CDCl}_{3}\right) \delta: 172.01,160.07,135.88$, 129.53, 128.77, 128.14, 127.82, 127.70, 114.46, 113.86, 95.07, 77.34, 77.02, 76.70, 67.36, 64.18, 55.33, 53.67, 51.81.

MS $\mathrm{m} / \mathrm{z}\left(\mathrm{M}^{+}-\mathrm{NO}_{2}\right)$ 309(35), 250(100), 223(88).207(39), 147(47), 115(28).

EA required for $\mathrm{C}_{19} \mathrm{H}_{20} \mathrm{~N}_{2} \mathrm{O}_{5}$ : C 64.0, H 5.7, $\mathrm{N} 7.9 \%$; found: C 63.8, H 5.8, N 7.9\%.

(2S,3S,4R,5S)-Methyl 5-(4-fluorophenyl)-4-nitro-3phenylpyrrolidine-2-carboxylate (4q).

According to P2-AgOTf, pale yellow oil.

$[\alpha]_{\mathrm{D}}{ }^{20}=65.2\left(\right.$ c 1.2, $\mathrm{CHCl}_{3}$ ) 99:1er. HPLC (Daicel Chiralpak AD-H), 2-propanol/n-hexane 10:90, flow rate $1.0 \mathrm{~mL} / \mathrm{min}, t_{R}$ 16.9 min (minor), 19.4 min (major).

IR (neat) $v_{\max }: 1221,1509,1548,1736 \mathrm{~cm}^{-1}$.

${ }^{1} \mathrm{H}$ NMR $\delta: 2.62$ (br. s, $\left.1 \mathrm{H}, \mathrm{NH}\right), 3.30$ (s, $3 \mathrm{H}, \mathrm{OCH}_{3}$ ), 4.41 (dd, $J=8.2,8.2 \mathrm{~Hz}, 1 \mathrm{H} \mathrm{CHPh}), 4.50$ (d, $J=9.1 \mathrm{~Hz}, 1 \mathrm{H}, \mathrm{CHCO})$, 4.77 (d, $J=8.3 \mathrm{~Hz}, 1 \mathrm{H}, \mathrm{CHN}), 5.21$ (dd, $J=8.2,8.2 \mathrm{~Hz}, 1 \mathrm{H}$, $\mathrm{CHNO}_{2}$ ), 7.07-7.16 (m, 2H, ArH), 7.24-7.38 (m, 5H, $\mathrm{ArH}$ ), 7.56-7.62 (m, 2H, $\mathrm{ArH})$.

${ }^{13} \mathrm{C}$ NMR $\delta: 51.8(\mathrm{CHPh}), 53.4\left(\mathrm{OCH}_{3}\right), 64.0(\mathrm{CHCO}), 66.8$ $(\mathrm{CHN}), 94.9\left(\mathrm{CHNO}_{2}\right), 115.9,127.8,128.2,128.7,128.8$, 133.7, 135.7, 161.7, $164.2(\operatorname{ArC}), 171.9(C O)$.

MS m/z: $297\left(\mathrm{M}^{+}-\mathrm{NO}_{2}, 14\right), 238$ (74), 211 (100), 135 (23), 133 (20), 115 (21).

HRMS required for $\left(\mathrm{C}_{18} \mathrm{H}_{17} \mathrm{FN}_{2} \mathrm{O}_{4}-\mathrm{NO}_{2}\right)$ 297.1181; found: 297.1175 .

\section{(2S,3S,4R,5S)-Methyl 5-(4-bromophenyl)-4-nitro-3- phenylpyrrolidine-2-carboxylate (4r).}

According to P2-AgOTf, colorless prisms, $n$-hexane/Et ${ }_{2} \mathrm{O}$ 3:1. mp $149-151^{\circ} \mathrm{C}$.

$[\alpha]_{\mathrm{D}}{ }^{20}=66.4$ (c 1, $\mathrm{CHCl}_{3}$ ) 99:1 er. HPLC (Daicel Chiralpak AD-H), 2-propanol $/ n$-hexane $10: 90$, flow rate $1.0 \mathrm{~mL} / \mathrm{min}, \mathrm{t}_{\mathrm{R}}$ $23.6 \mathrm{~min}$ (minor), $25.5 \mathrm{~min}$ (major).

IR (solid) $v_{\max }: 1213,1547,1738 \mathrm{~cm}^{-1}$.

${ }^{1} \mathrm{H}$ NMR $\delta 2.73$ (br. s, 1H, NH), 3.31 (s, 3H, $\mathrm{OCH}_{3}$ ), 4.40 (dd, $J=8.7 \mathrm{~Hz}, 1 \mathrm{H}, \mathrm{CHPh}), 4.53$ (d, $J=9.1,1 \mathrm{~Hz}, \mathrm{CHCO}), 4.80$ (d, $J=8.4 \mathrm{~Hz}, 1 \mathrm{H}, \mathrm{CHN}), 5.23\left(\mathrm{dd}, J=8.4,8.4 \mathrm{~Hz}, 1 \mathrm{H}, \mathrm{CHNO}_{2}\right.$ ), 7.17-7.29 (m, 2H, ArH), 7.31-7.33 (m, 3H, ArH), 7.44-7.51 (m, 2H, $\mathrm{ArH})$, 7.55-7.61 (m, 2H, ArH).

${ }^{13} \mathrm{C}$ NMR $\delta: 52.0(\mathrm{CHPh}), 53.2\left(\mathrm{OCH}_{3}\right), 63.8(\mathrm{CHCO}), 66.4$ $(\mathrm{CHN}), 94.1\left(\mathrm{CHNO}_{2}\right), 123.3,127.7,127.8,128.9,129.4$, 132.3, 132.4, 134.9 (ArC), 171.4 (CO).

MS m/z:359 ( $\left.\mathrm{M}^{+}-\mathrm{NO}_{2}, 21 \%\right), 357$ (22), 300 (65), 298 (68), 273 (97), 271 (100), 219 (30), 218 (14), 197 (15), 195 (21), 192 (96), 191 (35), 115 (17), 114 (32).

HRMS required for $\left(\mathrm{C}_{18} \mathrm{H}_{17} \mathrm{BrN}_{2} \mathrm{O}_{4}-\mathrm{NO}_{2}\right)$ : 359.0343; found: 359.0334 .

EA required for $\mathrm{C}_{18} \mathrm{H}_{17} \mathrm{BrN}_{2} \mathrm{O}_{4}$ : C 53.3, H 4.2, $\mathrm{N}$ 6.9\%; found: C 52.9, H 4.0, N 6.8\%.

(2S,3S,4R,5S)-Methyl phenylpyrrolidine-2-carboxylate (4s).

According to P2-AgOTf, colorless prisms, $n$-hexane/Et ${ }_{2} \mathrm{O}$ 5:1. mp $76-78^{\circ} \mathrm{C}$.

$[\alpha]_{\mathrm{D}}{ }^{20}=46.0\left(\right.$ c 0.6, $\left.\mathrm{CHCl}_{3}\right)$ 92:8er.HPLC (Daicel Chiralpak AD-H), 2-propanol $/ n$-hexane 10:90, flow rate $1.0 \mathrm{~mL} / \mathrm{min}, \mathrm{t}_{\mathrm{R}}$ 19.9 min (minor), 23.0 min (major).

IR (solid) $v_{\max }: 1550,1743 \mathrm{~cm}^{-1}$.

${ }^{1} \mathrm{H}$ NMR $\delta: 2.83$ (br. s, $\left.1 \mathrm{H}, \mathrm{NH}\right), 3.33$ (s, $3 \mathrm{H}, \mathrm{OCH}_{3}$ ), 4.46 (dd, $J=8.7,8.7 \mathrm{~Hz}, 1 \mathrm{H} \mathrm{CHPh}), 4.60$ (d, $J=9.1 \mathrm{~Hz}, 1 \mathrm{H}, \mathrm{CHCO})$, 5.02 (d, $J=8.5 \mathrm{~Hz}, 1 \mathrm{H}, \mathrm{CHNaph),} 5.41$ (dd, $J=8.4,8.4 \mathrm{~Hz}$, $1 \mathrm{H}, \mathrm{CHNO}_{2}$ ), 7.26-7.36 (m, 5H, ArH), 7.51-7.53 (m, 2H, $\operatorname{ArH}), 7.73$ (dd, $J=8.5,1.8 \mathrm{~Hz}, 1 \mathrm{H}, \operatorname{Ar} H), 7.72-7.9$ (m, 4H, $\operatorname{Ar} H)$.

${ }^{13} \mathrm{C}$ NMR $\delta: 52.0(\mathrm{CHPh}), 53.5\left(\mathrm{OCH}_{3}\right), 64.0$ (CHCO), 67.4(CHNf), $94.1\left(\mathrm{CHNO}_{2}\right), 123.9,126.7,127.8,128.2,128.4$, 128.5, 128.6, 128.9, 129.3, 129.4, 133.2, 133.6, 134.9, 135.9 (ArC), $171.8(C O)$.

MS m/z: $329\left(\mathrm{M}^{+}-\mathrm{NO}_{2}, 38 \%\right), 271$ (19), 270 (88), 244 (20), 243 (100), 227 (14), 167 (34), 165 (27), 115(26).

HRMS required for $\left(\mathrm{C}_{22} \mathrm{H}_{20} \mathrm{~N}_{2} \mathrm{O}_{4}\right)$ 376.1442, found: 376.1432 . EA required for $\mathrm{C}_{22} \mathrm{H}_{20} \mathrm{~N}_{2} \mathrm{O}_{4}$ : C 70.2, H 5.4, N 7.4\%; found C 69.8, H 5.5, N 7.4\%.

\section{References and Notes}

$\diamond$ This work has been supported by the Spanish Ministerio de Ciencia e Innovación (MICINN) (Consolider INGENIO 2010 CSD2007-00006, CTQ2010-20387) and Ministerio de Economía y Competitvidad (MINECO) (CTQ2013-43446P, CTQ2014-51912-REDC), FEDER, Generalitat Valenciana (PROMETEO/2009/039 and 2014/017), and by the University of Alicante. L. M. C. thanks the MICINN for a FPI fellowship. Financial support was also provided by the MICINN and FEDER (projects CTQ2010-16959/BQU, the University of the Basque Country (UPV/EHU, UFI11/22QOSYC)and the Basque Government (GV/EJ, grant IT-324-07). The authors thank the SGI/IZO-SGIker UPV/EHU and the DIPC for generous allocation of computational resources. 
(1) For general reviews dealing with general 1,3-DC, see: (a) Synthetic Applications of 1,3-Dipolar Cycloaddition Chemistry Towards Heterocycles and Natural Products, Padwa, A.; Pearson, W. H. Eds. John Wiley \& Sons, New Jersey, 2003. (b) Nájera, C.; Sansano, J. M. Curr. Org. Chem.2003, 7, 1105. (c) Eberbach, W. Sci. Synth. 2004, 27, chp. 11, 441. (d) Coldham, I.; Hufton, R. Chem. Rev. 2005, 105, 2765.(e) Nair, V.; Suja, T. D. Tetrahedron2007, 63, 12247. (f) Padwa, A.; Bur, S. K. Tetrahedron2007, 63, 5341. (g) Kanemasa, S. Heterocycles 2010, 82, 87.(h) Han, M.-Y.; Jia, J.-Y.; Wang, W. Tetrahedron Lett. 2014, 55, 784.

(2) Nájera, C.; Sansano, J. M. Curr. Top. Med. Chem. 2014, $14,1271$.

(3) (a) Zubia, A.; Mendoza, L.; Vivanco, S.; Aldaba, E.; Carrascal, T.; Lecea, B.; Arrieta, A.; Zimmerman, T.; Vidal-Vanaclocha, F.; Cossío, F. P. Angew. Chem. Int. Ed. 2005, 44, 2903. (b) San Sebastián, E.; Zimmerman, T.; Zubia, A.; Vara, Y.; Martín, E.; Sirockin, F.; Dejaegere, A.; Stote, R. H.; López, X.; Pantoja-Uceda, D.; Valcárcel, M.; Mendoza, L.; Vidal-Vanaclocha, F.; Cossío, F. P.; Blanco, F. J. J. Med. Chem. 2013, 56, 735.

(4) Tripathi, R. P.; Bisht, S. S.; Pandey, V. P.; Pandey, S. K.; Singh, S.; Sinha, S. K.; Chatuvedi, V. Med. Chem. Res. 2011,20, 1515.

(5) Conde, E.; Bello, D.; de Cózar, A.; Sánchez, M.; Vázquez, M. A.; Cossío, F. P. Chem. Sci.2012, 3, 1486.

(6) For recent reviews of asymmetric 1,3-DC, see: (a) Pellissier, H.Tetrahedron2007, 63, 3235. (b) Nájera, C.; Sansano J. M. in Topics in Heterocyclic Chemistry, vol. 12 (Ed.: HassnerA.), Springer-Verlag: Berlin-Heidelberg, 2008, pp. 117. (c) Stanley, L. M.; Sibi, M. P.Chem.Rev.2008, 108, 2887; (d) Álvarez-Corral,M.; Muñoz-Dorado, M.; Rodríguez-García, I. Chem. Rev. 2008, 108, 3174; (e) Naodovic, M.; Yamamoto, H. Chem. Rev. 2008, 108, 3132; (f) Nájera, C.; Sansano, J. M.; Yus, M. J. Braz. Chem. Soc.2010, 21, 377; (g) Kissane, M.; Maguire, A. R. Chem. Soc. Rev. 2010, 39, 845; (h) Adrio, J.; Carretero, J. C. Chem. Commun. 2011, 47, 6784.

(7) The exo descriptor refers the approacch of the dipolarophile in which the electron-withdrawing group of the 1,3-dipole and the nitro group are orientated in the opposite direction.

(8) Arai, T.; Mishiro, A.; Yokoyama, N.; Suzuki, K.; Sato, H. J. Am. Chem. Soc. 2010, 132, 5338.

(9) Yan, X.-X.; Peng, Q.; Zhang, Y.; Hong, W.; Hou, X.-L.; Wu, Y.-D. Angew. Chem. Int. Ed. 2006, 45, 1979.

(10) Grigg, R.; Kilner, C.; Sarker, M. A. B.; Orgaz de la Cierva, C.; Dondas, H. A. Tetrahedron2008, 64, 8974.

(11) Silver catalyzed processes were attempted obtaining low diastereoselections and moderate to low ee: (a) Shimizu, K.; Ogata, K.; Fukuzawa, S.-I. Tetrahedron Lett. 2010, 51, 5068. (b) Gu, X.; Xu, Z.-J.; Lo, V. K.-Y.; Che, C.-M. Synthesis 2012, 44, 3307.

(12) Imae, K.; Konno, T.; Ogata, K.; Fukuzawa, S.-I. Org. Lett. 2012, 14, 4410.

(13) Teichert, J. F.; Feringa, B. L. Angew. Chem. Int. Ed.2010, 49, 2486.
(14) Phosphoramidite ligands $\mathbf{1}$ are considered as a family of the second-generation privileged ligands: a) http://www.sigmaaldrich.com; ChemFiles2006, 6, 7; b) Zhou, Q.-L. In Privileged Chiral Ligands and Catalysts; Wiley-VCH; Weinheim, 2011.

(15) For an account, see: Nájera, C.; Sansano, J. M. J. Organomet. Chem.2014, 771, 78.

(16) Castelló, L. M.; Nájera, C.; Sansano, J. M.; Larrañaga, O.; de Cózar, A.; Cossío, F. P. Org. Lett.2013, 15,2902; Synfacts2013, 9, 0985.

(17) Castelló, L. M.; Nájera, C.; Sansano, J. M.; Larrañaga, O.; de Cózar, A.; Cossío, F. P. Adv. Synth. Catal.2014, 356,3861.

(18) (a) Nájera, C.; Retamosa, M. G.; Sansano, J. M. Angew. Chem. Int. Ed. 2008, 47, 6055; (b) Nájera, C.; Retamosa, M. G.; Martín Rodríguez, M.; Sansano, J. M.; de Cózar, A.; Cossío, F. P. Eur. J. Org. Chem.2009, 15, 5622.

(19) de Cózar, A. Private communication. 